\title{
3D acoustic propagation through an estuarine salt wedge at low-to-mid-frequencies: Modeling and measurement
}

D. Benjamin Reeder, and Ying-Tsong Lin

Citation: The Journal of the Acoustical Society of America 146, 1888 (2019); doi: 10.1121/1.5125258

View online: https://doi.org/10.1121/1.5125258

View Table of Contents: https://asa.scitation.org/toc/jas/146/3

Published by the Acoustical Society of America

\section{ARTICLES YOU MAY BE INTERESTED IN}

Introduction to the special issue on three-dimensional underwater acoustics

The Journal of the Acoustical Society of America 146, 1855 (2019); https://doi.org/10.1121/1.5126013

Underwater acoustic energy fluctuations during strong internal wave activity using a three-dimensional parabolic equation model

The Journal of the Acoustical Society of America 146, 1875 (2019); https://doi.org/10.1121/1.5125260

Measurements and modeling of acoustic propagation in a scale model canyon

The Journal of the Acoustical Society of America 146, 1858 (2019); https://doi.org/10.1121/1.5125130

Beam tracing for two- and three-dimensional problems in ocean acoustics

The Journal of the Acoustical Society of America 146, 2016 (2019); https://doi.org/10.1121/1.5125262

Three-dimensional bottom diffraction in the North Pacific

The Journal of the Acoustical Society of America 146, 1913 (2019); https://doi.org/10.1121/1.5125427

Source triangulation utilizing three-dimensional arrivals: Application to the search for the ARA San Juan submarine

The Journal of the Acoustical Society of America 146, 2104 (2019); https://doi.org/10.1121/1.5125251

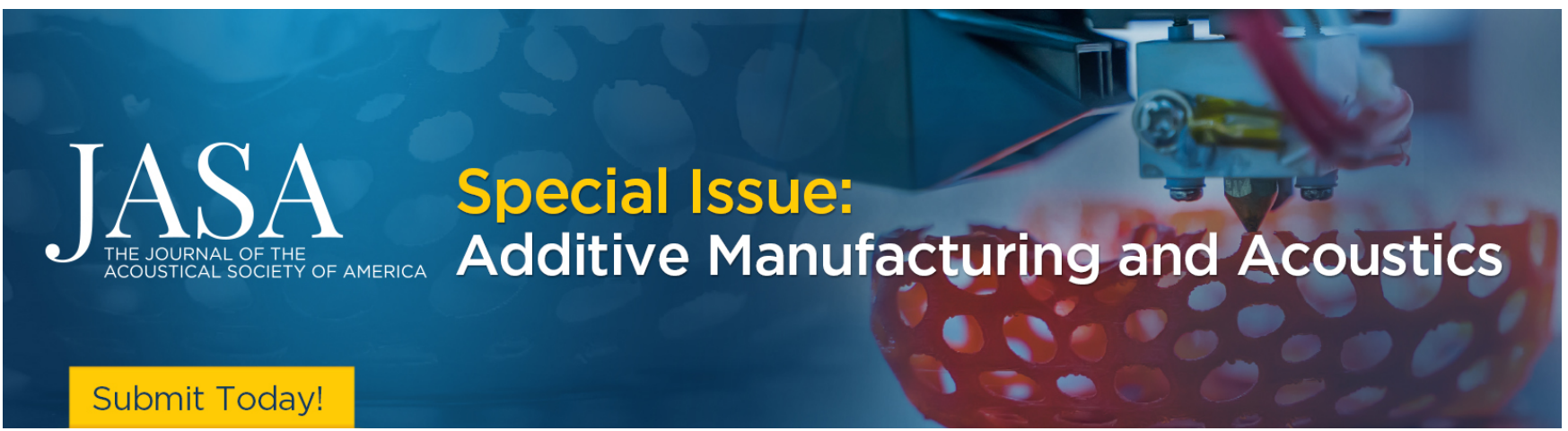




\title{
3D acoustic propagation through an estuarine salt wedge at low-to-mid-frequencies: Modeling and measurement
}

\author{
D. Benjamin Reeder ${ }^{a)}$ \\ Department of Oceanography, Naval Postgraduate School, 833 Dyer Road, Monterey, California 93943, USA \\ Ying-Tsong Lin \\ Applied Ocean Physics \& Engineering Department, Woods Hole Oceanographic Institution, \\ 213 Bigelow Laboratory, MS\#11, Woods Hole, Massachusetts 02543, USA
}

(Received 31 December 2018; revised 31 May 2019; accepted 6 June 2019; published online 1 October 2019)

\begin{abstract}
The estuarine salt wedge presents a dynamic and highly refractive waveguide, the acoustic propagation characteristics of which are controlled by the water column sound speed gradient and boundary interactions. Acoustically, the salt wedge consists of two isospeed layers separated by a thin, threedimensional (3D), high-gradient layer. The behavior of a broadband $(500-2000 \mathrm{~Hz})$ acoustic field under the influence of an estuarine salt wedge in the Columbia River estuary is explored using two $3 \mathrm{D}$ acoustic propagation models: 3D rays and 3D parabolic equation. These model results are compared to data collected during the field experiment. Results demonstrate that the dominant physical mechanism controlling acoustic propagation in this waveguide shifts from 3D bottom scatter in a non-refractive waveguide (before the entrance of the salt wedge) to 3D acoustic refraction with minimal bottom interaction in a refractive waveguide (when the salt wedge occupies the acoustic transect). Vertical and horizontal refraction in the water column and out-of-plane scattering by the bottom are clearly evident at specific narrowband frequencies; however, these mechanisms contribute to, but do not account for, the total observed broadband transmission loss.
\end{abstract} https://doi.org/10.1121/1.5125258

[WLS]

Pages: 1888-1902

\section{INTRODUCTION}

An estuary is a semi-enclosed coastal body of water which has a free connection with the open sea and within which sea water is measurably diluted with fresh water derived from land drainage (Pritchard, 1967). A more complete description is offered by Day (1980, 1981) and Kennish (1986) as "a partially enclosed coastal body of water which is either permanently or periodically open to the sea and within which there is a measureable variation of salinity due to the mixture of seawater with freshwater derived from land drainage." Estuaries can be classified according to the geophysical processes by which they are formed [e.g., coastal plain or drowned river estuaries, fjord or glacier-built estuaries, bar-built or lagoon-type estuaries, and tectonic estuaries (Pritchard, 1967)], by their water column properties (e.g., salt wedge, partially mixed, well-mixed, inverse, and intermittent), by their salinity and tidal characteristics (e.g., salinity zonation, tidal range), by their sedimentation, or by their ecosystem energetics (Kennish, 1986). During rising tides, the water column structure of salt wedge estuaries consists of denser seawater advected under fresh water discharged by the river. The degree of water column stratification is controlled by the tide's range and speed of advance, river discharge volumetric flow rate, and river mouth morphology (Dyer, 1998). Investigations of near- and in-shore environments have naturally focused on geological, thermodynamic, and hydrodynamic parameters of the estuarine/riverine environment. The relatively

${ }^{\text {a)} E l e c t r o n i c ~ m a i l: ~ d b r e e d e r @ n p s . e d u ~}$ few acoustical studies carried out in rivers, estuaries, or other energetic environments have employed higher frequencies at downward-looking orientations [e.g., water column echosounder surveys, multibeam echosounder (swath) bottom surveys]. The objective of this line of investigation is to explore threedimensional (3D) acoustic propagation characteristics of the estuarine waveguide to facilitate a more complete understanding of the physical environment.

Acoustically, this environment consists of two isospeed layers separated by a thin, range- and depth-dependent, highgradient layer comprising the salt wedge interface. When the fresh water is warm relative to the salt water, the competing effects of temperature and salinity on sound speed may create a sound speed gradient too small to cause acoustic refraction. When the fresh water is cold relative to the salt water, both temperature and salinity will contribute to create a stronger sound speed gradient compared to that observed during summer. While this nominally three-layer, very shallow water acoustic waveguide is dominated by high-angle multipath propagation at very short ranges, it has been shown that refraction occurring in the gradient layer supports ducting of low-angle energy in the upper layer, and that the statistical variability of acoustic signal increases due to the spatiotemporal dynamics of the salt wedge structure as it advances and retreats on the rising and falling tides (Reeder, 2016).

A small acoustical field experiment was carried out in May 2013 in the Mouth of the Columbia River (MCR) within the context of a larger hydrodynamic field experiment entitled Rivers and Inlets II funded by the Office of Naval Research. Reeder (2016) reported details of the experiment, 
acoustic data analysis, and results of two-dimensional (2D) acoustic propagation modeling, confirming the hypothesis that the estuarine salt wedge is acoustically observable at low-to-mid frequencies $(500-2000 \mathrm{~Hz})$. The present work presents additional insights into the physics of the acoustic propagation based upon $3 \mathrm{D}$ acoustic modeling, with the primary goal of establishing the contribution of horizontal refraction to total transmission loss (TL) and impulsive arrival structures.

The paper is organized as follows: Section II gives an overview of the experiment and data analysis results to provide context for the 3D modeling results, Sec. III provides a description of the models used in the present work, Sec. IV includes acoustic propagation modeling results and discussion, and Sec. V presents a concise summary.

\section{OVERVIEW OF EXPERIMENT, DATA ANALYSIS, AND MODELING}

\section{A. Columbia River estuary environment}

The Columbia River estuary is classified as partially mixed (Neal, 1972) and mesotidal (Sherwood and Creager, 1990), possessing mixed diurnal and semidiurnal tides with a mean tidal excursion of approximately $2 \mathrm{~m}$ (Neal, 1972) and maximum observed tidal excursion as great as $3.4 \mathrm{~m}$ during spring tides (Gelfenbaum, 1983). This field experiment occurred during the highly energetic spring freshet when the river was stratified (i.e., possessed a salt wedge), and experienced strong tidal currents, large river volumetric flow rates, and rough surface conditions. The experimental area spanned from several kilometers west of the North Jetty and South
Jetty at the river mouth to points in the North Channel (Fig. 1). During flood, the turbulent convergence of the advancing ocean tide against the strong river outflow generated a salt wedge, the boundary of which is $3 \mathrm{D}$ (forming a horse-shoe shaped surface expression in the center of the channel between the mouth and Jetty A with the fresh river water advancing westward toward the mouth on the north and south sides), extended from the riverbed to the surface, and was highly dynamic, as evidenced by confused, breaking waves at the surface and down-welling at the front between the two water masses. During ebb, surface currents exceeded $3.5 \mathrm{~m} / \mathrm{s}$ in a direction opposing the predominant wind and swell direction, generating a rough surface condition. During both flood and ebb, strong bottom currents suspended and transported bottom sediment [predominantly fine sand with a mean grain size of $2.75 \phi(0.149 \mathrm{~mm})$ (Sherwood and Creager, 1990)], generating bedforms having amplitudes between 0.7 and $2.1 \mathrm{~m}$ and wavelengths between 20 and $90 \mathrm{~m}$ (Gelfenbaum et al., 2014, 2015). Bedforms are readily evident in Fig. 1 in both the northern and southern channels, as well as in the area immediately east of Station A5. The tidal currents in the estuary are strong, but highly variable on a tidal and sub-tidal timescales (Sherwood and Creager, 1990); therefore, the expectation is that the bedforms are not static, but are continuously changing during the acoustic observation period. The spatiotemporally varying 3D salt wedge shape, the strong interfacial gradient capable of hosting internal waves, the subduction of bubbles by down-welling at the front, the rough surface condition, and the rough, active riverbed are all acoustically significant features which conspire to create a very complicated and dynamic acoustic waveguide.

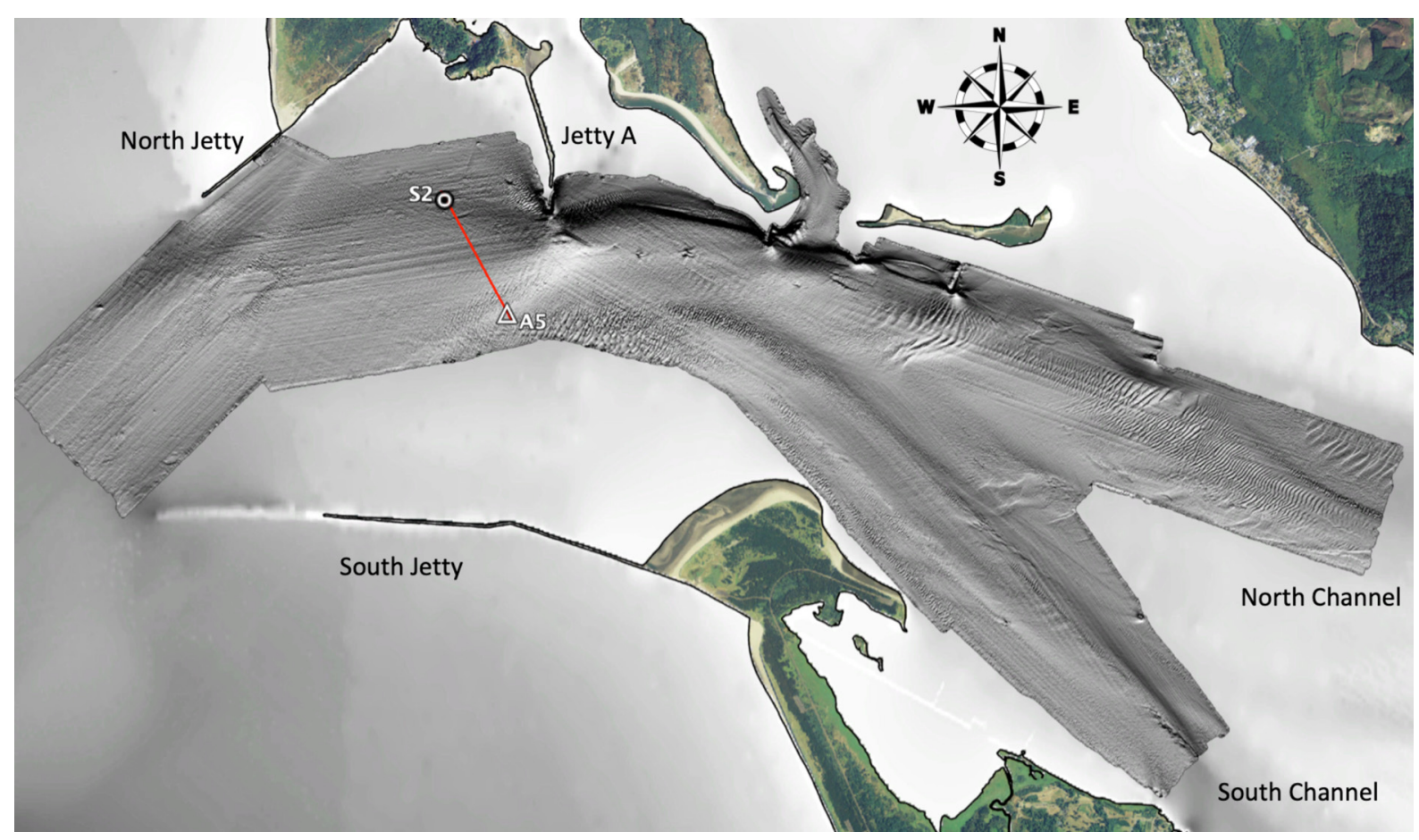

FIG. 1. (Color online) MCR with high-resolution bathymetry: The acoustic source was deployed at Station S2 and the receiver was deployed $1 \mathrm{~m}$ above the riverbed at Station A5. The acoustic transect was $1.36 \mathrm{~km}$ long, oriented NNW-SSE and centered on $4615.528^{\prime} \mathrm{N}, 1242.354^{\prime} \mathrm{W}$. Bedform amplitudes were $0.7-2.1 \mathrm{~m}$ and wavelengths were $20-90 \mathrm{~m}$. The entire displayed area is $8.5 \mathrm{~km} \times 15 \mathrm{~km}$. Map data: SIO, NOAA, U.S. Navy, NGA, GEBCO, USGS, Google 2018. From Gelfenbaum et al. (2015). 


\section{B. Data collection, analysis, and 2D modeling}

Hydrophones, temperature and pressure sensors, and acoustic current meters were moored at multiple locations throughout the experimental area. Conductivity Temperature Depth (CTD) casts were conducted throughout using a Castaway CTD (Sontek, San Diego, CA). The data from which were provided to the Center for Coastal Margin Observation and Prediction (CMOP) in Portland, OR for validation of their river forecast model, predictions from which were used as environmental input to acoustic propagation modeling (discussed in Sec. III).

The present work focuses on the period of the procession of the salt wedge (flood) on May 27, 2013 during which acoustic signals (2-s long, linear frequency-modulated in the $500-2000 \mathrm{~Hz}$ band) were transmitted at a constant source level once every $10 \mathrm{~s}$ by an acoustic source (LL-1424HP, Lubell Labs, Whitehall, $\mathrm{OH}$ ) at $7 \mathrm{~m}$ water depth at Station $\mathrm{S} 2$ and received by a hydrophone (Acousonde, Greeneridge Sciences, Santa Barbara, CA) $1 \mathrm{~m}$ above the riverbed at Station A5 (Fig. 1). This $1.36 \mathrm{~km}$ long acoustic transect, transverse to the direction of salt wedge movement, was strategically chosen to provide the optimal geometry to observe the refractive properties of the salt wedge. The received signals were matched-filtered in the frequency domain, increasing temporal resolution and signal-to-noise ratio. The signal intensity level (SIL) is defined as

$$
\mathrm{SIL}=10 \log \left[\int_{0}^{T} p^{2}(t) d t\right] \mathrm{dB} \text { re } 1 \mu \mathrm{Pa}^{2}-\mathrm{s},
$$

where $p(t)$ is the matched-filter output and $T$ is the integration period equal to $0.5 \mathrm{~s}$.

Figure 2 (taken from Reeder, 2016) presents data collected during flood on May 27. The top panel shows sound speed as a function of depth (m) and range (m) along the acoustic path between the source at Station S2 and the receiver at Station A5. This across-channel transect is shown looking from west to east into the estuary as the salt wedge enters the estuary. Using the Chen-Millero equation (Chen and Millero, 1977), the sound speed is converted from temperature and salinity profiles provided by CMOP's integrated modeling system named the "Virtual Columbia River," which is based upon a distributed network of multiple data sources (Baptista et al., 2015; Karna et al., 2015). The source is depicted on the left at $7 \mathrm{~m}$ water depth and the receiver is depicted on the right $1 \mathrm{~m}$ above the riverbed. The CMOP model output was provided in $36 \mathrm{~s}$ increments, and this particular profile is the one for $1915 \mathrm{Z}$ on May 27, which is the approximate time at which the acoustic source occupies the depth of greatest sound speed gradient. The low resolution bathymetry was extracted from the United States Geological Survey (USGS) multi-beam echosounder bathymetric surveys conducted in 2012 and provided by CMOP. Water depth varies from $16 \mathrm{~m}$ at Station S2 to a maximum of $27 \mathrm{~m}$ in the center of the channel, back up to $17 \mathrm{~m}$ at Station A5; modeled sound speed varies from a minimum of $1460 \mathrm{~m} / \mathrm{s}$ in the near-surface waters to a maximum sound speed of $1490 \mathrm{~m} / \mathrm{s}$ in the lower portion of the water column.
[These modeled sound speed fields were used in Reeder (2016), but they were not used in the 3D calculations in this paper.] The middle panel shows sound speed $(\mathrm{m} / \mathrm{s})$ as a function of depth $(\mathrm{m})$ from CTD profiles collected on a Castaway CTD hand-deployed over the side of the ship in approximately $15-\mathrm{min}$ intervals during the 6-h acoustic transmission period from $1530 \mathrm{Z}$ to $2130 \mathrm{Z}$ on May 27. Sound speed is observed to vary between 1460 and $1490 \mathrm{~m} / \mathrm{s}$ primarily due to the salinity variation of $28 \mathrm{psu}$, while temperature varied by only $1.5^{\circ} \mathrm{C}$. Note that as the salt wedge advances during flood, $1925 \mathrm{Z}$ is the approximate time at which the source at $7 \mathrm{~m}$ water depth moves from fresh water to salt water. The bottom panel shows 1-min averaged relative broadband (BB) SIL in $\mathrm{dB}$ re $1 \mu \mathrm{Pa}^{2} \mathrm{~s}$ (normalized to the maximum BB SIL during the 6-h observation period) observed at Station A5 vs time on the same scale as shown in the middle panel. The BB SIL is relatively stable prior to $1700 \mathrm{Z}$, slowly decreases approximately $15 \mathrm{~dB}$ during a $21 / 2$ hour period, reaches a minimum at approximately 1925Z, then rapidly increases $15 \mathrm{~dB}$ during a $1 \mathrm{~h}$ period, finally returning at $2030 \mathrm{Z}$ to approximately the same level as observed prior to $1700 \mathrm{Z}$. Variability in BB SIL during the period of decreasing and increasing BB SIL ( 1730Z-2030Z) is greater than the variability at the beginning and end of the transmission period.

Reeder (2016) attributed the behavior of TL to vertical and horizontal refraction by the higher speed water as the salt wedge crossed the acoustic transect between S2 and A5: as the salt water progressively fills the water column along the acoustic transect, an increasing amount of acoustic energy is directed vertically up and horizontally out of plane to the east, away from the acoustic receiver. The maximum TL occurs when the sound speed gradient and acoustic source are at the same depth ( 1915-1930Z), resulting in the greatest degree of acoustic refraction of the entire period. This acoustic feature is bracketed before and after by isospeed (non-refractive) water. This interpretation was supported by acoustic modeling, but the Bellhop2D model (Porter and Bucker, 1987; Porter, 2011) employed underpredicted the change in TL, presumably because it could not account for horizontal refraction in the acoustic field. The remainder of this paper employs 3D acoustic models to explore the propagation physics and to quantify the out-of-plane bottom scatter, diffraction, and horizontal refraction caused by the $3 \mathrm{D}$ salt wedge.

\section{ACOUSTIC PROPAGATION MODELS}

Predictions by two 3D acoustic propagation models are presented to elucidate the behavior of the acoustic field under the influence of the salt wedge: the 3D ray model and the three-dimensional parabolic equation (3DPE) model.

\section{A. Descriptions of acoustic models}

The ray model directly solves the $3 \mathrm{D}$ eikonal equation $|\nabla \tau|^{2}=c^{-2}(\vec{x})$, resulting from the ray-series expansion of the Helmholtz wave equation and taking only the first-order term for high frequencies (Jensen et al., 1994). In the eikonal equation, $\tau$ is the time advance term in the phase of the plane-wave solution, $\exp (i \omega \tau), \omega$ is the angular frequency, and $c(\vec{x})$ is the 3D sound speed field. Actually, $\nabla \tau$ indicates 

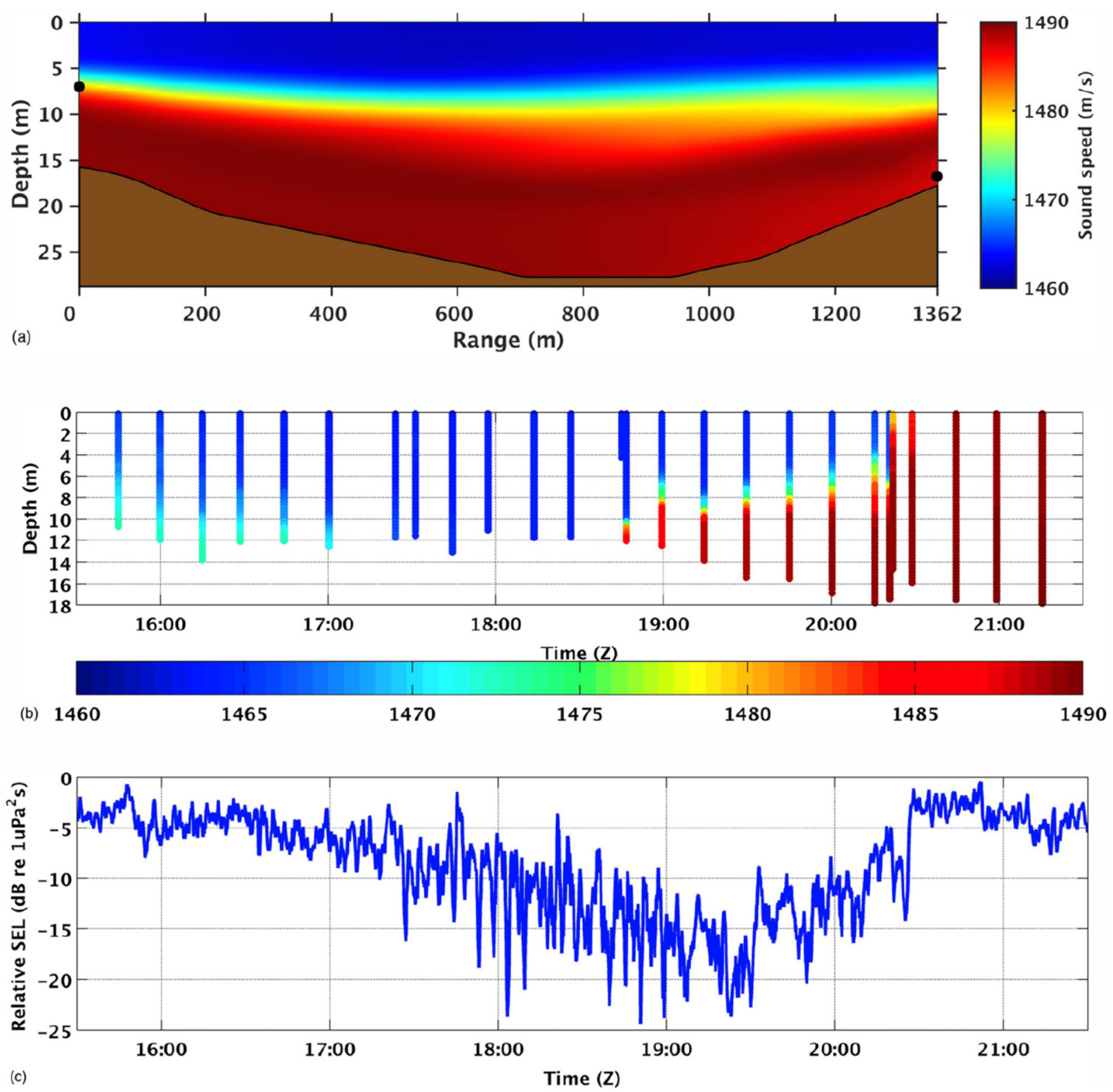

FIG. 2. (Color online) (a) Modeled sound speed (m/s) vs depth (m) and range (m) along the S2-A5 acoustic transect at model time 1915Z on May 27, 2013. The solid circle on the left marks the position of the acoustic source $7 \mathrm{~m}$ below the surface. The solid circle on the right marks the position of the acoustic receiver $1 \mathrm{~m}$ above the riverbed at Station A5. (Modeled sound speed courtesy of CMOP.) (b) Sound speed (m/s) vs depth (m) and time ( $Z$ ) at Station S2 based on CTD casts during the $6 \mathrm{~h}$ period of the acoustic transmissions shown in the lower panel. (c) Relative broadband signal intensity level (dB re $1 \mu$ Pa $\left.{ }^{2} \mathrm{~s}\right)$ vs time ( $Z$ ) observed at Station A5 on May 27. All three panels from Reeder (2016).

directions of ray trajectories (the normal of the wavefront), and the eikonal equation governs its length $|\nabla \tau|$, leading to the following second-order ordinary differential equation (ODE) for tracing rays with given initial positions and launching angles

$$
\frac{d}{d s}\left(\frac{1}{c} \frac{d \vec{x}}{d s}\right)=-\frac{1}{c^{2}} \nabla c,
$$

where $s$ is the arc-length along the ray. The MATLAB ${ }^{\circledR}$ ODE45 toolbox was utilized to solve the ray-tracing ODE in a $3 \mathrm{D}$ Cartesian coordinate system, and the law of reflection is enforced on the sea surface and sea floor. The $3 \mathrm{D}$ ray model was validated by comparison to the analytical solutions for constant and linear sound speed profiles.
The second model is a 3DPE model (Lin et al., 2013) that implements the Split-Step Fourier (SSF) algorithm to solve the approximated Helmholtz wave equation of parabolic type for sound pressure, which is not resolved by the $3 \mathrm{D}$ ray model. The general parabolic-equation approximation method was first introduced by Hardin and Tappert (1973) and Tappert (1974) to the underwater acoustic community, and it has been shown to be one of the most efficient and effective numerical methods to predict underwater sound propagation in a complex environment. In the present 3D SSF parabolic equation (PE) model, a density-reduced pressure variable $\tilde{P}=P / \sqrt{\rho}$ (Bergman, 1946) was used to handle the density $(\rho)$ variation across the seafloor interface, and the approximated Helmholtz equation of parabolic type 
can be written as follows in a 3D Cartesian coordinate system:

$$
\begin{aligned}
& \frac{\partial}{\partial x} \psi(x, y, z) \\
& =i k_{0}\left\{-1+\sqrt{k_{0}^{-2}\left(\frac{\partial^{2}}{\partial y^{2}}+\frac{\partial^{2}}{\partial z^{2}}\right)+\tilde{n}^{2}(x, y, z)}\right\} \\
& \quad \times \psi(x, y, z)
\end{aligned}
$$

where $\psi$ is the demodulated sound pressure with the baseline phase removed according to the reference wavenumber $k_{0}$ $=\omega / c_{0}$ [i.e., $\tilde{P}=\psi \exp \left(i k_{0} x\right)$ ], and $\tilde{n}$ is the effective index of refraction taking into account the density variation across the seafloor interface,

$$
\tilde{n}=\frac{k^{2}}{k_{0}^{2}}+\frac{1}{2 k_{0}^{2}}\left[\frac{1}{\rho} \nabla^{2} \rho-\frac{3}{2 \rho^{2}}(\nabla \rho)^{2}\right] .
$$

The key step of PE methods is to approximate the square root operator in Eq. (3); the following wide-angle approximation proposed by Feit and Fleck (1978) is utilized in the present 3D SSF PE model:

$$
\begin{aligned}
& \sqrt{k_{0}^{-2}\left(\frac{\partial^{2}}{\partial y^{2}}+\frac{\partial^{2}}{\partial z^{2}}\right)+\tilde{n}^{2}(x, y, z)} \\
& =\sqrt{k_{0}^{-2}\left(\frac{\partial^{2}}{\partial y^{2}}+\frac{\partial^{2}}{\partial z^{2}}\right)+1}+\tilde{n}(x, y, z)-1 .
\end{aligned}
$$

The solution of Eq. (3) with the wide-angle approximation can be determined using the SSF algorithm (Lin et al., 2013),

$$
\begin{aligned}
\psi(x+\Delta x, y, z)= & e^{i k_{0} \Delta x(n-1)} \\
& \times F^{-1}\left\{e^{i \Delta x\left[-k_{0}+\sqrt{k_{0}^{2}-k_{y}^{2}-k_{z}^{2}}\right]} F\{\psi(x, y, z)\}\right\},
\end{aligned}
$$

where $F$ and $F^{-1}$ indicate 2D Fourier transform and inverse transform to transfer the PE solution between the spatial domain $(y, z)$ and the wavenumber domain $\left(k_{y}, k_{z}\right)$ at each marching step. Between the transforms, $e^{i \Delta x\left[-k_{0}+\sqrt{k_{0}^{2}-k_{y}^{2}-k_{z}^{2}}\right]}$ is the free space propagator which is numerically operated in the wavenumber domain. After this SSF step, the phase of the PE pressure solution is adjusted according to the anomaly of the index of refraction through $e^{i k_{0} \Delta x(n-1)}$ in the spatial domain. Detailed descriptions of the 3D SSF PE are contained in Lin et al. (2013).

\section{B. Environmental input to acoustic models}

Three environments were used as input into the acoustic propagation models: an idealized salt wedge with lowresolution $(80 \mathrm{~m}$ ) bathymetry (Environment 1$)$, an idealized salt wedge with high-resolution ( $1 \mathrm{~m}$ ) bathymetry (Environment 2 ), and a more realistic environment using CMOP modeled temperature and salinity fields and high-resolution bathymetry
(Environment 3). While more realistic, the variability and uncertainty of the CMOP environmental fields created difficulty in the identification of trends in the acoustic field; hence, the idealized, well-behaved salt wedge (Fig. 3) provided additional insight into the physics of the acoustic propagation. Characteristics of the top and bottom boundaries of the waveguide were shared by all three environments. In both acrossand along-path directions, the top boundary (water surface) was horizontally planar (range independent), while the bottom boundary was rough (range-dependent bathymetry), yet rangeindependent in terms of geoacoustic parameters of the riverbed. The riverbed was modeled using the high-resolution $(1 \mathrm{~m})$ bathymetry in Fig. 3, extracted from a subset of the USGS survey (Fig. 4) performed in the weeks following the acoustic field experiment (Gelfenbaum et al., 2014, 2015). The geoacoustic parameters in Environments 2 and 3 associated with the predominantly fine sandy sediment with mean grain size of $2.75 \phi$ $(0.149 \mathrm{~mm})$ were a sound speed $(c)$ of $1620 \mathrm{~m} / \mathrm{s}$, density $(\rho)$ of $1.83 \mathrm{~g} / \mathrm{cm}^{3}$, and attenuation $(\alpha)$ of $0.365 \mathrm{~dB} / \mathrm{m}$ (Sherwood and Creager, 1990; Zhou et al., 2009).

\section{Idealized salt wedge}

Figure 3 presents the salt wedge from the perspective of an observer looking south-to-north across the river along the acoustic transect from the receiver to the source as the salt wedge advances into the river from the west during flood. The idealized salt wedge consisted of a two-layer water column: an upper fresh water layer (with a sound speed of $1460 \mathrm{~m} / \mathrm{s}$ ) separated from the lower salt water layer (with a sound speed of $1490 \mathrm{~m} / \mathrm{s}$ ) by an interface characterized by a $4 \mathrm{~s}^{-1}$ vertical sound speed gradient and a $4^{\circ}$ slope (in the direction of the river axis). These two characteristics represent the observed sound speed gradient and slope, based upon the CTD observations. The water column sound speed field was range-dependent in the across-path direction and

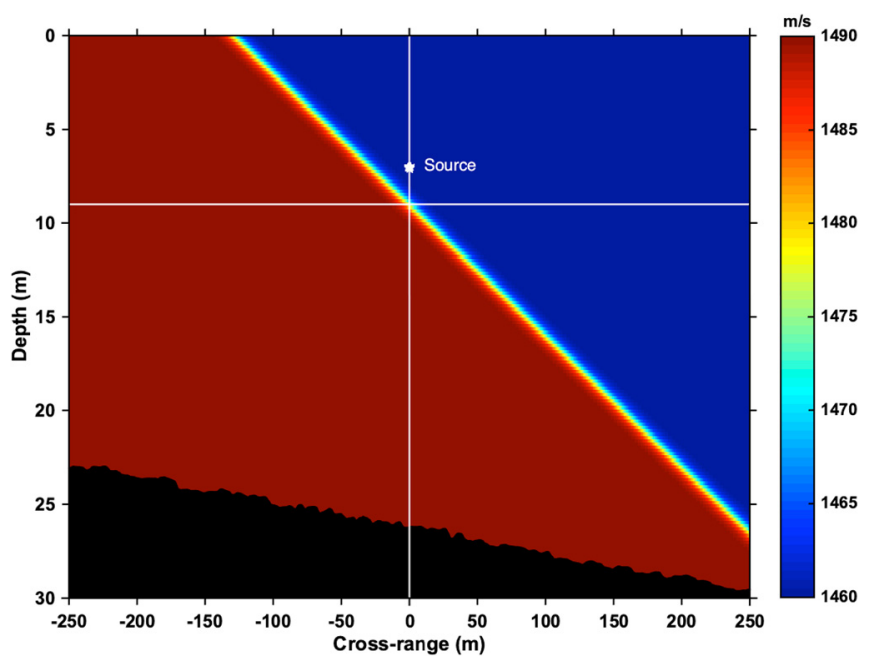

FIG. 3. (Color online) Idealized salt wedge environment, looking south-tonorth across the river along the acoustic transect from the receiver to the source as the salt wedge advances into the river from the west during flood; the salt wedge, with $41 / \mathrm{s}$ gradient and $4^{\circ}$ slope, separates salt water $(1490 \mathrm{~m} / \mathrm{s})$ and fresh water $(1460 \mathrm{~m} / \mathrm{s})$. Environment $2 \mathrm{~b}$ is depicted here, with the source at $7 \mathrm{~m}$ water depth, $2 \mathrm{~m}$ above the gradient and $18 \mathrm{~m}$ above the riverbed [USGS high resolution $(1 \mathrm{~m})$ bathymetry]. 


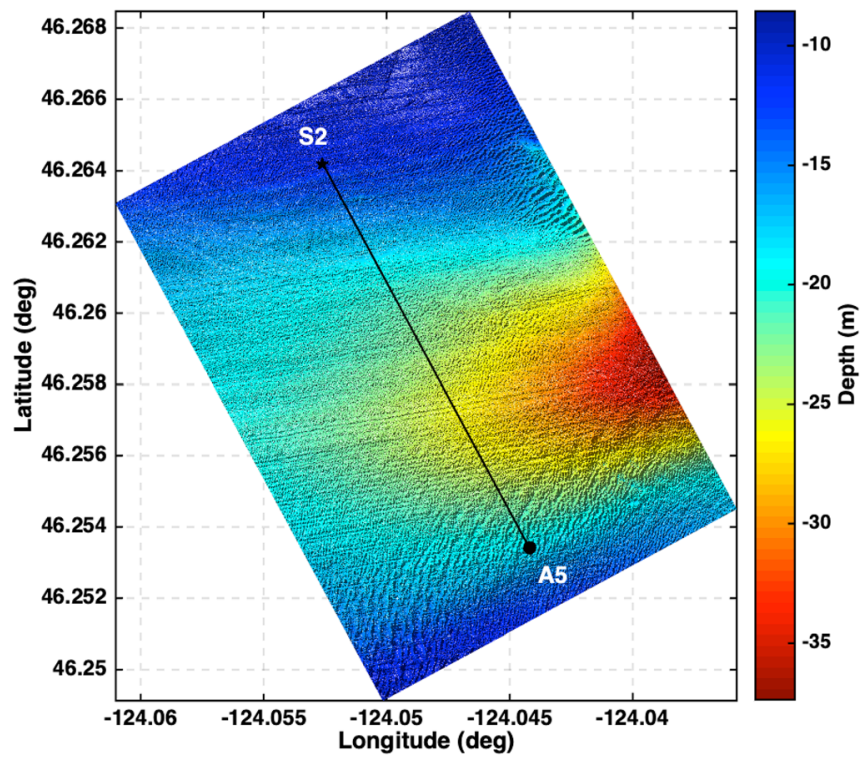

FIG. 4. (Color online) High-resolution $(1 \mathrm{~m})$ bathymetry along the acoustic track between the source (S2) and receiver (A5). The riverbed slopes down from west to east and hosts bedforms which are oriented at angles oblique to the acoustic transect. Bathymetric data courtesy USGS.

range-independent in the along-path direction. Environment 1 was used in the $3 \mathrm{D}$ ray model, and Environment 2 was used in the PE acoustic propagation model. The lowresolution bathymetry was derived from the high-resolution bathymetry.

\section{Realistic salt wedge}

Environment 3 consisted of sound speed fields converted and interpolated from 234 gridded temperature and salinity profiles provided by CMOP's integrated modeling system and was used in the PE acoustic propagation model. The modeled water column gradient is known to be weaker than actual (Geyer, 2014); therefore, the sound speed gradient at the salt wedge interface in Environment 3 was increased to approximately $4 \mathrm{~s}^{-1}$ to more closely represent the actual, observed gradient.

\section{RESULTS AND DISCUSSION}

\section{A. 3D ray predictions}

Two realizations of Environment 1 are used in this section: The first realization (Environment 1a) is a completely isospeed fresh water environment of $1460 \mathrm{~m} / \mathrm{s}$ to represent a non-refractive medium of the river prior to the entrance of the salt wedge; the second realization (Environment $1 \mathrm{~b}$ ) represents the time during flood (approximately $3 \mathrm{~h}$ later) at which the acoustic source occupies a depth just above the salt wedge gradient. Figures 5 and 6 display results from the $3 \mathrm{D}$ ray model for Environments $1 \mathrm{a}$ and $1 \mathrm{~b}$, respectively. Panel (a) displays the sound speed field as a function of depth $[Z(m)]$ and cross-range $[Y(m)]$ halfway between the source at S2 and the receiver at A5 looking from the south as the salt wedge advances across the acoustic transect from left to right. Panel (b) displays the sound speed field as a
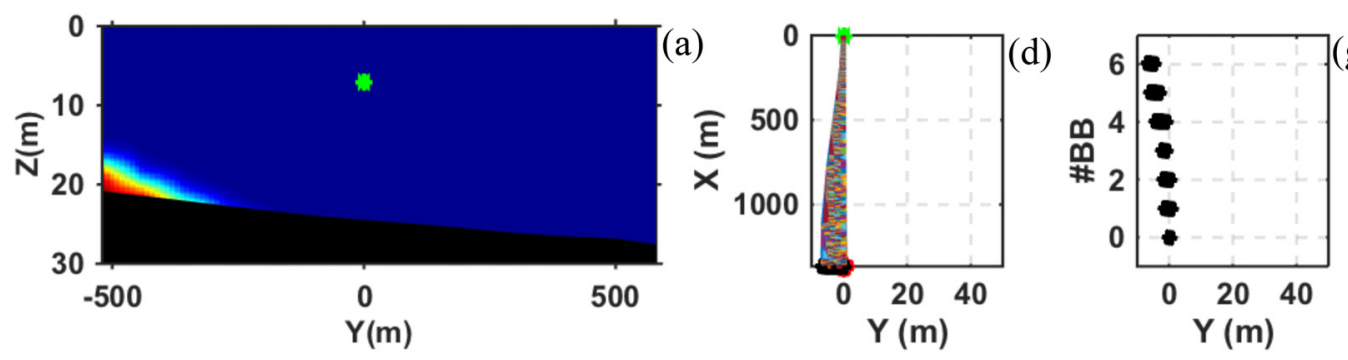

(g)

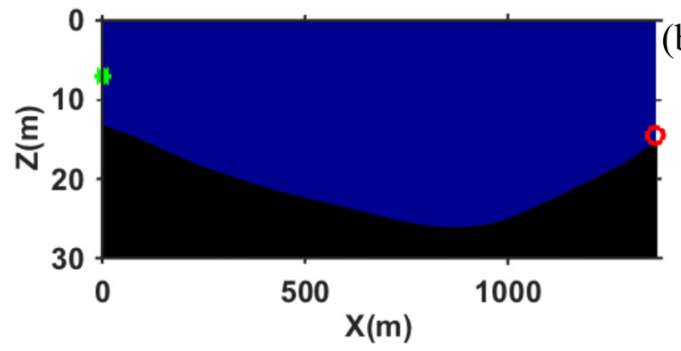

(b)
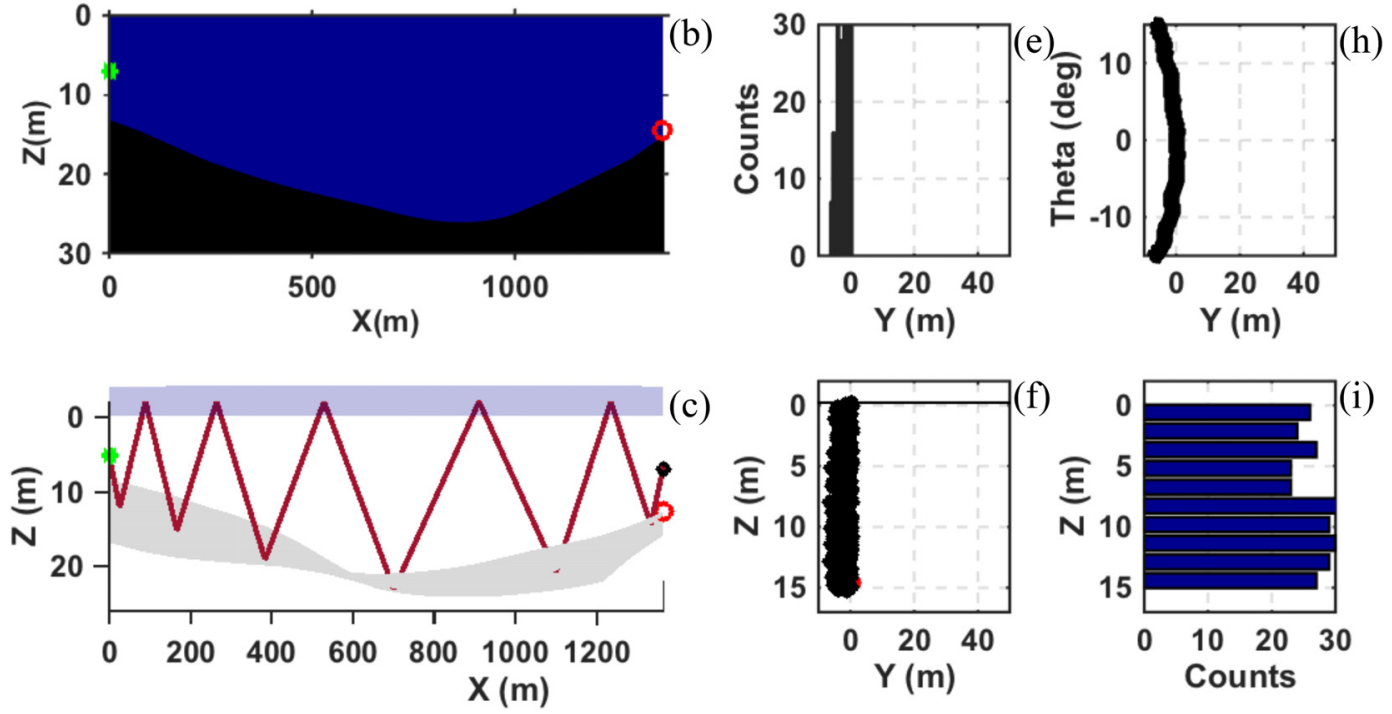

FIG. 5. (Color online) Modeled 3D ray results using Environment 1a. This environment is a non-refractive, isospeed waveguide representing early flood as the salt wedge is just beginning to enter the waveguide from the left/west. (a)-(c) depict the acoustic waveguide; (e)-(i) are visualizations of the rays at the end of the acoustic transect. 

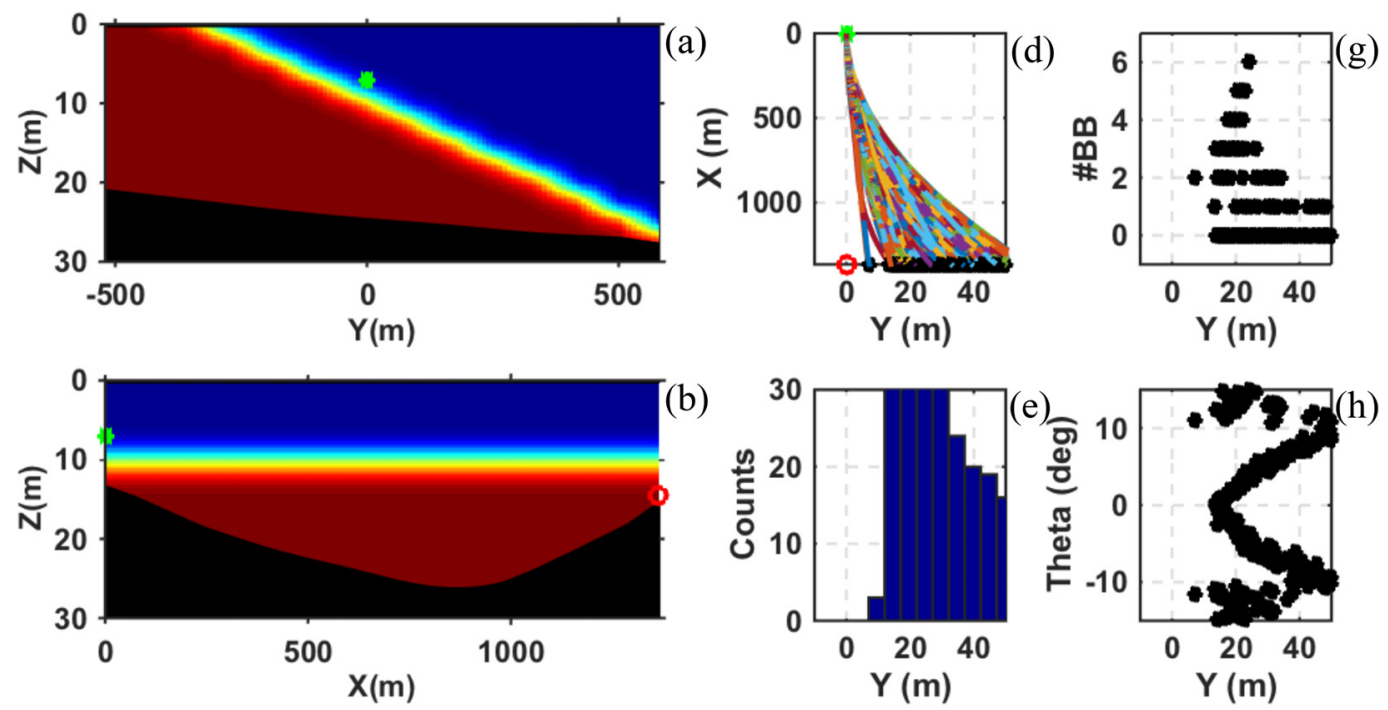

b)
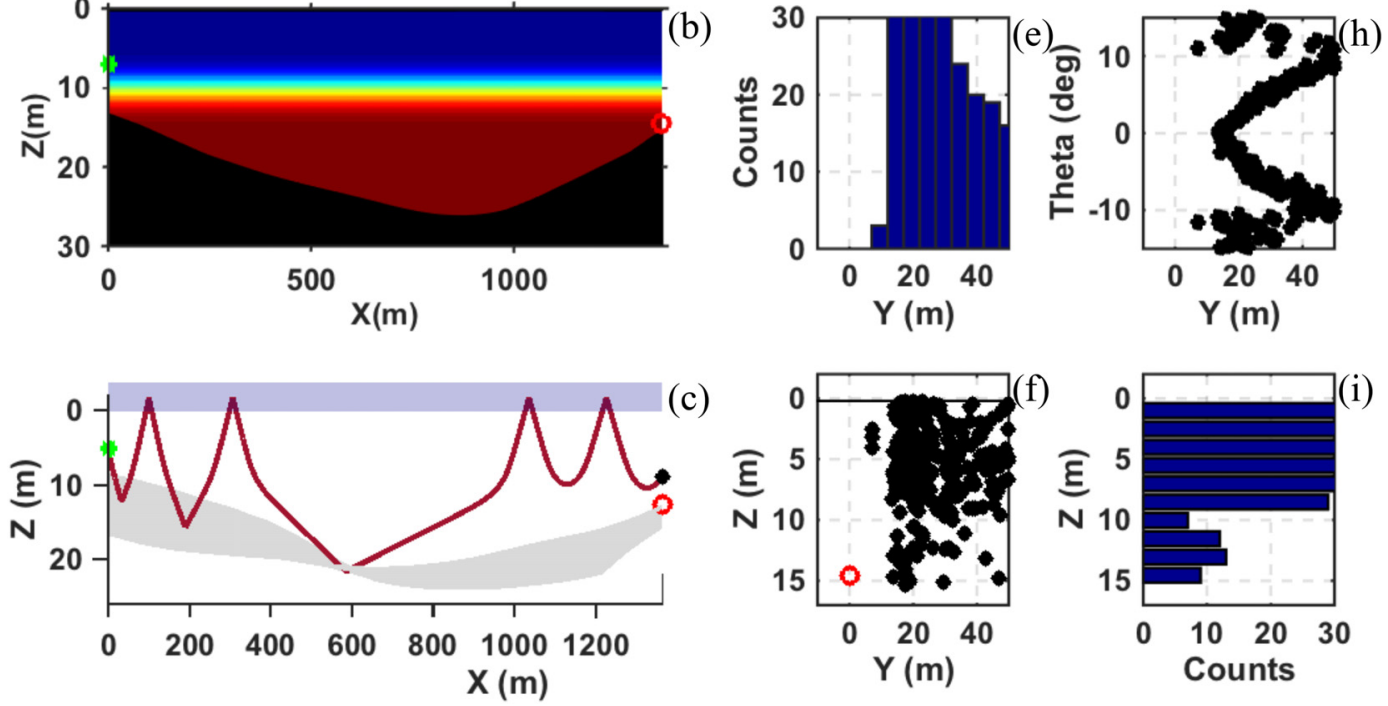

FIG. 6. (Color online) Modeled 3D ray results using Environment $1 \mathrm{~b}$. This environment is a highly refractive waveguide representing mid-flood as the salt wedge gradient crosses the acoustic transect near the depth of the source. Plots similar to Fig. 5.

function of depth $[Z(m)]$ and range $[X(m)]$ between the source at S2 (left) and the receiver at A5 (right) looking from the west, as the salt wedge gradient rises to more shallow depths as it progressively fills the water column during flood. Panel (c) shows rays as a function of depth $[Z(m)]$ and range $[X(m)]$ from selected launch angles interacting with the surface and riverbed (gray shaded area depicts the top of the sediment layer) as the energy propagates from source to receiver. Note that the slope of the riverbed changes near the center of the channel-it initially slopes to the west (downriver), then slopes to the east (upriver) after $r=600 \mathrm{~m}$. Panel (d) plots the rays as a function of range $[X(m)]$ and cross-range $[Y(m)]$ as they propagate from the source (top) to the receiver (bottom). Panels (e)-(i) are visualizations of the rays at the end of the acoustic transect. Panel (e) is a histogram of cross-range $[Y(m)]$ deflection. Panel (f) plots the landing points in depth $[Z(m)]$ and cross-range $[Y(m)]$ of those rays that reach the end of the acoustic transect. Panel ( $\mathrm{g}$ ) displays the number of bottom bounces encountered by the rays as a function of cross-range $[Y(m)]$. Panel $(\mathrm{h})$ presents launch angle $(\theta$, in degrees relative to the horizontal) as a function of cross-range $[Y(m)]$. Finally, panel (i) is a histogram of vertical $[Z(m)]$ displacement of the rays. In all panels, the green circle indicates the location of the source and the red circle indicates the location of the receiver. Also in all cases, the vertical launch angles of the rays vary between $\pm 15^{\circ}$, and the azimuthal launch angle (in the crossrange direction) is zero.

Figure 5 shows results for Environment 1a, representing early flood, before the acoustic field begins to interact with the salt wedge gradient. The salt wedge is seen approaching from the west (left) in Fig. 5(a) but has yet to cross the acoustic transect in Fig. 5(b). Figure 5(c) shows one steepangled ray, experiencing no observable refraction by the isospeed water column. Figure 5(d) shows all of the rays plotted nearly on top of each other due to the lack of refraction in this isospeed environment; however, there is a small amount of spread between rays at the end of the transect. Many of the rays land on the downstream side of A5 as a result of the sloping bottom scattering the rays out-of-plane to the west. This indicates that the bottom slope along the first half of the transect is steeper than the bottom slope along the second half of the transect. Figures 5(e) and 5(f) demonstrate uniform distribution of energy throughout the water column, centered on a point approximately $5 \mathrm{~m}$ west of A5, as shown in Fig. 5(d). Figures 5(g) and 5(h) are consistent with Fig. 5(d), showing cross-range deflection of the rays to the west, while also highlighting the fact that the steepest angled rays experience the greatest out-of-plane scatter due to a larger number of bottom interactions along the transect. Figure 5(i), consistent with Figs. 5(e) and 5(f), demonstrates uniform distribution of energy throughout the water column.

As the salt wedge continues to move toward the acoustic transect, the sound speed field is progressively dominated by the strong sound speed gradient of the salt wedge. The advancing gradient causes the acoustic energy to refract vertically up away from the bottom and horizontally to the east (upriver), away from the receiver, with fewer and fewer rays interacting with the bottom. The point of maximum TL 
occurs as the gradient encounters the source, represented by Environment $1 \mathrm{~b}$ (Fig. 6). Figures 6(a) and 6(b) show the position of the salt wedge gradient relative to the source. Figure 6(c) shows one ray at a steep launch angle; rays interact with the bottom at the steepest angles only while all other rays remain waterborne in the surface duct. Figures $6(d)-6(h)$ show that all of the rays land to the east (upriver) of the receiver $[\mathrm{Y}(\mathrm{m}) \gg 0$ ]. In this particular case, the maximum horizontal deflection was approximately $Y(m)=80 \mathrm{~m}$ (not shown in the plot). Figure 6(g) shows that most of the rays do not encounter the riverbed $(\# \mathrm{BB}=0)$, but are trapped in the freshwater duct above the salt wedge. Figure 6 (h) shows that the salt wedge gradient has the greatest influence upon rays with launch angles near $\pm 10^{\circ}$; rays at the smallest launch angles are trapped in the freshwater duct and rays at the greatest launch angles spend more time at depths with weaker gradients. Figures 6(e), 6(f), and 6(i) show the non-uniform distribution of energy in the waveguide- the energy is refracted up $[\mathrm{Z}(m)>0]$ and to the east $[\mathrm{Y}(m)>0]$ of the receiver.

A visual comparison of ray-based model results in Figs. 5 and 6 demonstrate that the progressively refractive properties of the water column decrease the amount of acoustic energy interacting with the riverbed, consistent with Reeder (2016). Significantly, the dominant physical mechanism controlling TL shifts from bottom scatter in a non-refractive waveguide (before the entrance of the salt wedge) to $3 \mathrm{D}$ acoustic refraction with minimal bottom interaction in a refractive waveguide (when the salt wedge occupies the acoustic transect). Having examined this idealized environment using ray theory, Sec. IV B first presents PE model results using Environment 2 (Figs. 7-10) followed by results using Environment 3 (Figs. 11-12) in order to quantify the relative contributions of vertical and horizontal refraction and bottom scatter, and to confirm this interpretation by comparison to data.

\section{B. 3DPE modeling}

\section{Narrowband predictions for Environment 2}

For each time-dependent realization of the environmental model input, the PE acoustic model computes the acoustic field as well as the time series of the signal that reaches the end of the transect, from which the integrated energy is derived in terms of BB SIL (dB). Two realizations of Environment 2 are used in this section: The first realization (Environment $2 \mathrm{a}$, similar to Environment 1a and Fig. 5) is a completely isospeed fresh water environment of $1460 \mathrm{~m} / \mathrm{s}$ to represent a nonrefractive medium of the river prior to the entrance of the salt wedge; the second realization (Environment $2 \mathrm{~b}$, similar to Environment $1 \mathrm{~b}$ and Fig. 6) represents the time at which the acoustic source, at $7 \mathrm{~m}$ water depth, is $2 \mathrm{~m}$ above the salt wedge gradient (as shown in Fig. 3). Results using these two realizations of Environment 2 are presented in Figs. 7-10 for a frequency $(1250 \mathrm{~Hz})$ in the middle of the experimental band $(500-2000 \mathrm{~Hz})$ in terms of SIL (dB re $\left.1 \mu \mathrm{Pa}^{2} \mathrm{~s}\right)$ as a function of range $[x(m)]$ along the acoustic transect, and cross-range $[y(m)]$ in the top three panels and depth $[z(m)]$ in the bottom panels. The top three panels present a horizontal cross-section of SIL at 4, 8, and $12 \mathrm{~m}$, respectively; the bottom panels present a vertical cross-section of SIL along the acoustic transect.

Figure 7 shows the SIL in a non-refractive waveguide (Environment 2a), exhibiting a uniformly distributed acoustic field, disturbed only by interaction with the anisotropic bathymetry. The predominant riverbed slope is down from west to east which directs energy out-of-plane to the east while the rough bathymetry scatters energy both west and east of the transect. In contrast to the non-refractive isospeed medium in Fig. 7, 3DPE results for Environment $2 \mathrm{~b}$ are presented in Fig. 8. The 3D refraction is readily evident in all four panels resulting from the acoustic signal's interaction with the anisotropic water column sound speed field. Energy refracts to the east in the top three panels (upwards in the plots), modulated by the constructive and destructive interference of energy scattered by the bottom, generating beams of high-intensity acoustic energy propagating to the southeast, consistent with the refraction seen in the $3 \mathrm{D}$ ray predictions shown in Fig. 6. Strong depth-dependent shadow zones are also generated directly in front of the depth-dependent salt wedge gradient. Directly above the source at $7 \mathrm{~m}$, the shadow zones are strongest in terms of acoustic field intensity variation, while growing weaker in intensity but wider in spatial extent as a function of increasing depth. In the vertical (bottom panel), significant acoustic energy is trapped in the fresh water duct above the salt wedge. This is a snapshot in time; as the salt wedge advances upriver, the acoustic field shifts continuously, generating a high degree of acoustic variability at the receiver. Figure 9 shows $N \times$ two-dimensional parabolic equation (2DPE) results for the same environment as Fig. 8 (Environment 2 b), approximating the contribution to the acoustic field by vertical refraction alone. The spatial structure of the $N \times 2 \mathrm{DPE}$ acoustic field is similar to the 3DPE results in Fig. 8; however, the constructive and destructive interference patterns are weaker and less distinct, the shadow zones are weaker, and the acoustic field in the surface duct is less intense. The differences between the predicted 3DPE and $N \times 2$ DPE SIL are presented in Fig. 10. At short ranges, the differences are greatest in the riverbed due to the fact that the 3DPE model accounts for the energy that is scattered from in-plane to out-of-plane and from out-ofplane back into-plane as the energy propagates down-range (bottom panel). This bottom-interacting energy then contributes to the differences seen primarily in the lower portion of the water column at short ranges. Once the signal has a chance to interact sufficiently with the water column sound speed gradient, the differences increase with range throughout the water column, but with a high degree of spatial variability both in the horizontal (top three panels) and in the vertical (bottom panel). The differences in this simulation of coherent acoustic energy at this single frequency and single snapshot in time exceed $30 \mathrm{~dB}$ in some places in the waveguide (color scale limited to $\pm 10 \mathrm{~dB}$ for presentation purposes).

\section{Non-integrated BB predictions for Environment 3}

Figure 11 presents time-dependent $\mathrm{BB}$ SIL (dB re 1 $\mu \mathrm{Pa}^{2} \mathrm{~s}$ ) as a function of geo-time on the $y$ axis (hhmm) and travel time (msec) on the $x$ axis for Environment 3 during 

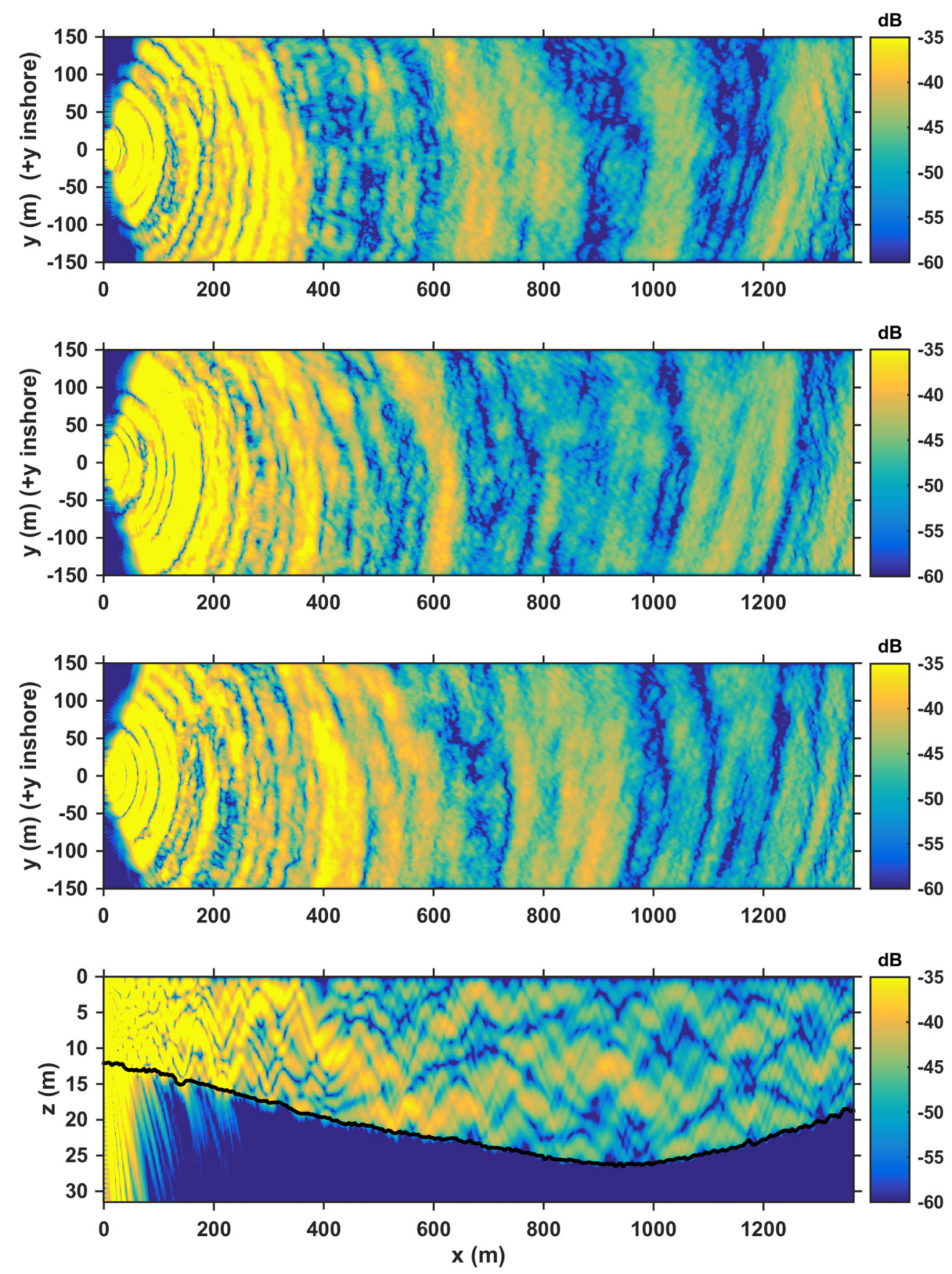

FIG. 7. (Color online) Modeled 3DPE SIL ( $\mathrm{dB}$ re $1 \mu \mathrm{Pa}^{2} \mathrm{~s}$ ) at $1250 \mathrm{~Hz}$ for a non-refractive, isospeed fresh water environment (Environment $\left.2 \mathrm{a}\right)$ as a function of range $[x(m)]$ along the acoustic transect and cross-range $[y(m)]$ in the top three panels and depth $[z(m)]$ in the bottom panel. The top three panels present a horizontal cross-section of SIL at 4, 8 , and $12 \mathrm{~m}$, respectively; the bottom panel presents a vertical cross-section of SIL along the acoustic transect.

the flood period in which the water column consisting of fresh water transitions to salt water; 2DPE arrival structures are on the left and the 3DPE arrivals structures are on the right, for points in the upper (top panels) and lower (bottom panels) portions of the water column at the end of the acoustic transect. The time series represents the arrival structure of the received $\mathrm{BB}$ energy resulting from multipath propagation in this waveguide.

These $2 \mathrm{D}$ vs $3 \mathrm{D}$ modeled arrival structures are similar in the overall structure, but there exist differences in the complexity of the arrival structures, particularly during the time the high sound speed gradient occupied depths near the source depth, consistent with the fact that the 3DPE model captures the contribution of the horizontally refracted energy to the constructive and destructive interference occurring during salt wedge passage. Additional observations that can be drawn from Fig. 11: (1) The travel time of the leading arrival through fresh water (top of each panel) is approximately $17 \mathrm{msec}$ greater than the travel time through salt water (bottom of each panel), consistent with a difference of approximately $25 \mathrm{~m} / \mathrm{s}$ in sound speed between the two water masses along the $1.36 \mathrm{~km}$ acoustic transect. (2) The arrival structures before and after salt wedge passage are not identical. The differences in structure can be attributed to the 

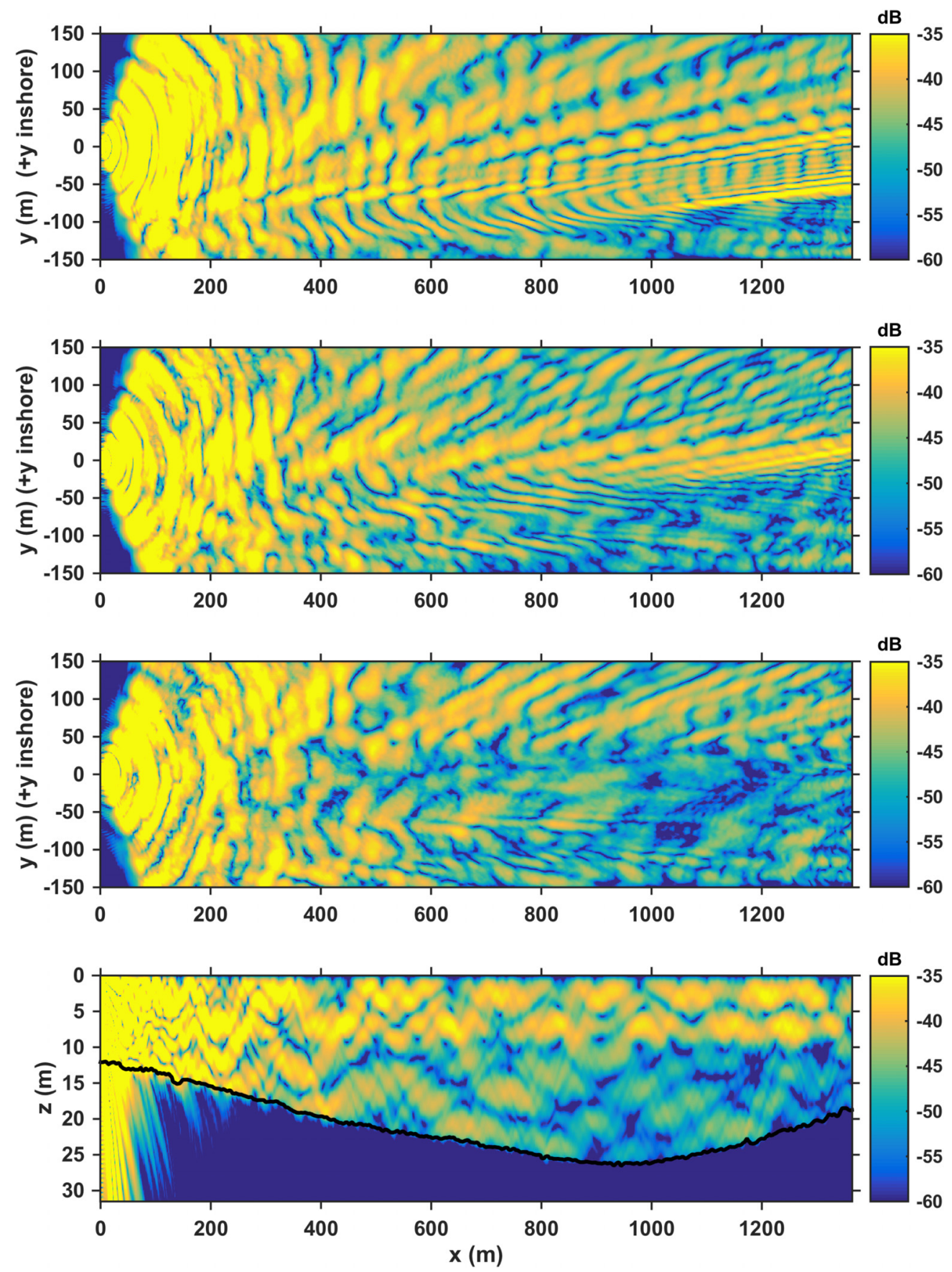

FIG. 8. (Color online) Modeled 3DPE SIL ( $\mathrm{dB}$ re $1 \mu \mathrm{Pa}^{2} \mathrm{~s}$ ) at $1250 \mathrm{~Hz}$ for the idealized salt wedge environment (Environment $2 \mathrm{~b}$ ) shown in Fig. 3. Plots are similar to Fig. 7. The non-uniform acoustic field in this refractive environment differs significantly from the acoustic field in the non-refractive environment shown in Fig. 7.

differences in fresh and salt water bottom reflection coefficients which vary with acoustic impedance at the water/bottom interface. Additionally, in this simulation using more realistic environmental input (Environment 3), some salt water lingers in the deepest parts of the riverbed channel at low tide, and fresh water is not completely removed from the entire transect at high tide. The differing acoustic impedances and residual inter-tidal salt and fresh water that creates only a nearly isospeed water column conspire to modify the interaction of the acoustic field with the riverbed that results in the non-identical arrival structures before and after salt wedge passage. (3) Both 2D and 3D models capture the dominant arrival in both the upper and lower water column, which arrive via lower angle refracted-refracted (RR) and refracted-surface reflected (RSR) paths. In the lower portion of the water column (bottom panels), the dominant arrival occurs primarily on the leading edge; however, in the upper water column, the dominant arrival is delayed during the period that the salt wedge occupies the acoustic transect and forms a freshwater surface duct. The dominant energy arriving via RR and RSR paths in the upper water column is delayed due to the slower-speed medium of the surface duct, while some weaker, more diffuse, earlier-arriving energy passes through the higher speed water of the salt wedge via 

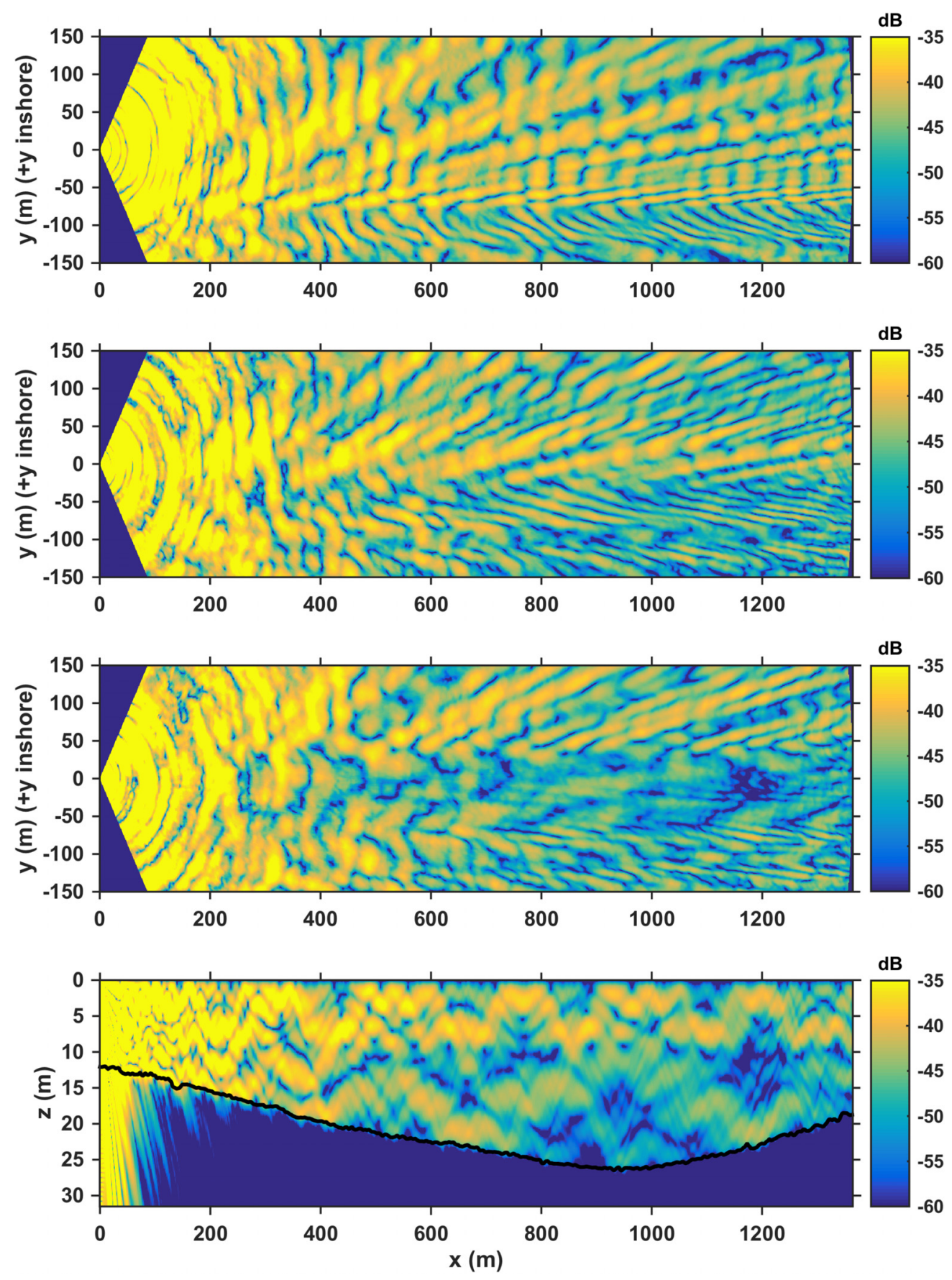

FIG. 9. (Color online) Modeled $N \times 2$ DPE SIL ( $\mathrm{dB}$ re $1 \mu \mathrm{Pa}^{2}$ s) at $1250 \mathrm{~Hz}$ for the idealized salt wedge environment (Environment 2b) shown in Fig. 3. Plots are similar to Fig. 7. The spatial structure of the acoustic field predicted here by $N \times 2 \mathrm{DPE}$ is similar to the acoustic field predicted by $3 \mathrm{DPE}$ in Fig. 8; however, the acoustic intensities differ significantly in some areas, as shown in Fig. 10.

lower-angle RR and refracted-bottom reflected (RBR) paths. (4) In the lower water column (bottom panels), the dominant arrival suffers degradation and largely disappears between $1925 \mathrm{Z}$ and 1935Z, when the salt wedge gradient encounters the source. This indicates that the dominant path is RR and that little to no energy arrives at the deeper receiver via the RR path. At this particular point in time, the signal encounters the greatest sound speed gradient, thus the greatest degree of refraction; a majority of the acoustic energy is strongly refracted to higher angles, scattering the energy to higher modes (steeper angles), resulting in maximum attenuation in the bottom per unit range. Whatever energy survives this highly refractive condition shows up as lower amplitude dispersed arrivals; the later arrivals during this period are weak or nonexistent. (5) The 3D results on the right more clearly represent the finer structure in the arrivals, and they include late-arriving energy from high-angle paths and outof-plane bottom scatter arriving via RBR and bottomreflected, surface-reflected paths which are not well represented by the 2D model. These finer elements and late arrivals are, however, much weaker than the dominant arrivals occurring earlier in time.

It is important to note that the similarities of the arrival structures before and after salt wedge passage and the 

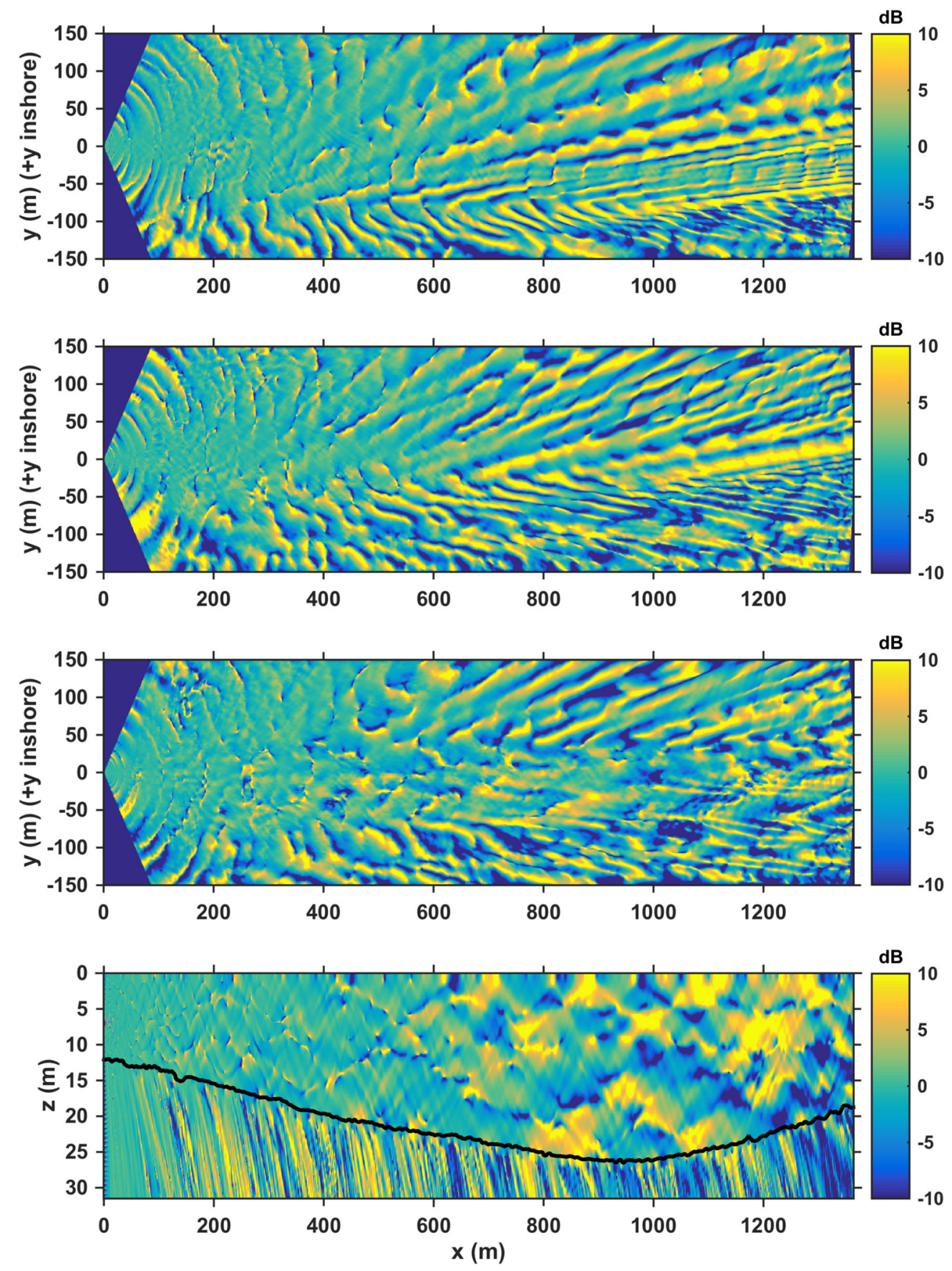

FIG. 10. (Color online) Modeled differences between 3DPE and $N \times 2 \mathrm{DPE}$ SIL (dB re $1 \mu \mathrm{Pa}^{2}$ s) at $1250 \mathrm{~Hz}$ for the idealized salt wedge environment (Environment 2b) shown in Fig. 3. Plots are similar to Fig. 7. The acoustic intensity differences increase with range, exceeding $30 \mathrm{~dB}$ in some places in the waveguide (color scale limited to $\pm 10 \mathrm{~dB}$ for presentation purposes).

weakness of the late arrivals indicate that the out-of-plane scattering and refraction captured by the 3D model is a real, but minor contributor to the total integrated BB energy received at the end of the acoustic transect. Incidentally, data-model comparisons of the arrival structures could not be performed; a prohibitively large amount of variability was present in the received signal due to a poor clock at the source, the movement of the source as it was suspended over the side of the ship on the free surface, and the dynamic water column and seabed during the transmissions. Future field work at MCR will include a precise clock and a moored source to facilitate an investigation of the observed arrival structures.

\section{Integrated BB predictions for Environment 3}

Figure 12 shows the same observed relative BB SIL during flood on May 27 as shown in Fig. 2(c) (thin blue solid line). The thick black solid line represents received $\mathrm{BB}$ acoustic energy predicted by Bellhop2D in terms of relative BB SIL received at the hydrophone at Station A5, from Reeder (2016). The Bellhop2D BB SIL shows a similar trend as the data: slowly varying but stable BB SIL prior to $1700 \mathrm{Z}$, slowly decreasing BB SIL during a $2 \frac{1}{2} \mathrm{~h}$ period to a minimum at approximately 1920Z, then rapidly increasing over a $1 \mathrm{~h}$ period to approximately the same level as 

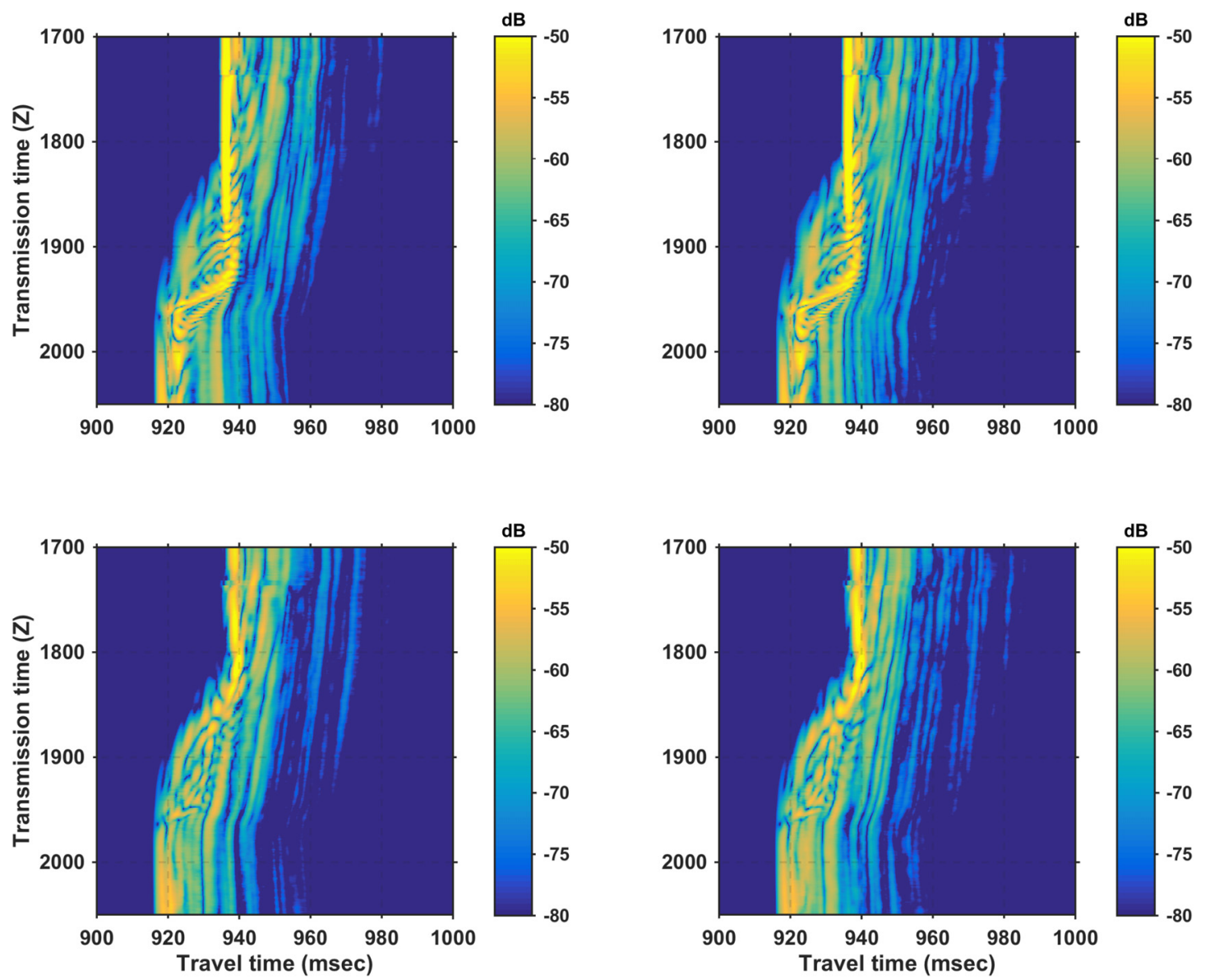

FIG. 11. (Color online) Modeled time-dependent PE BB SIL ( $\mathrm{dB}$ re $1 \mu \mathrm{Pa}^{2} \mathrm{~s}$ ) (“arrival structures") received at the end of the acoustic transect as a function of transmission time on the $y$ axis (hhmm) and travel time (msec) on the $x$ axis for Environment 3 during the flood period; 2DPE (left column) and 3DPE (right column) arrival structures for a point in the upper (top row) and lower (bottom row) portions of the water column at Station A5. The travel time difference between the top and bottom of each panel is $17 \mathrm{msec}$. The 3D results on the right more clearly represent the finer structure in the arrivals.

observed prior to $1700 \mathrm{Z}$. While showing similar trends, the Bellhop2D modeled BB SIL decreases by only $6 \mathrm{~dB}$ near $1720 \mathrm{Z}$, significantly less than the $15 \mathrm{~dB}$ decrease observed in the data. The thick black dotted line represents received acoustic energy predicted by 3DPE using Environment 3 in terms of relative BB SIL received at Station A5. The 3DPE
BB SIL was computed at approximately 1 min temporal resolution, $5 \mathrm{~Hz}$ spectral resolution, and range, cross-range, and depth spatial resolutions of $1,0.35$, and $0.175 \mathrm{~m}$, respectively, showing a similar trend as the data and the Bellhop2D predictions. The differences between 3DPE and Bellhop2D can be attributed to (a) environmental model

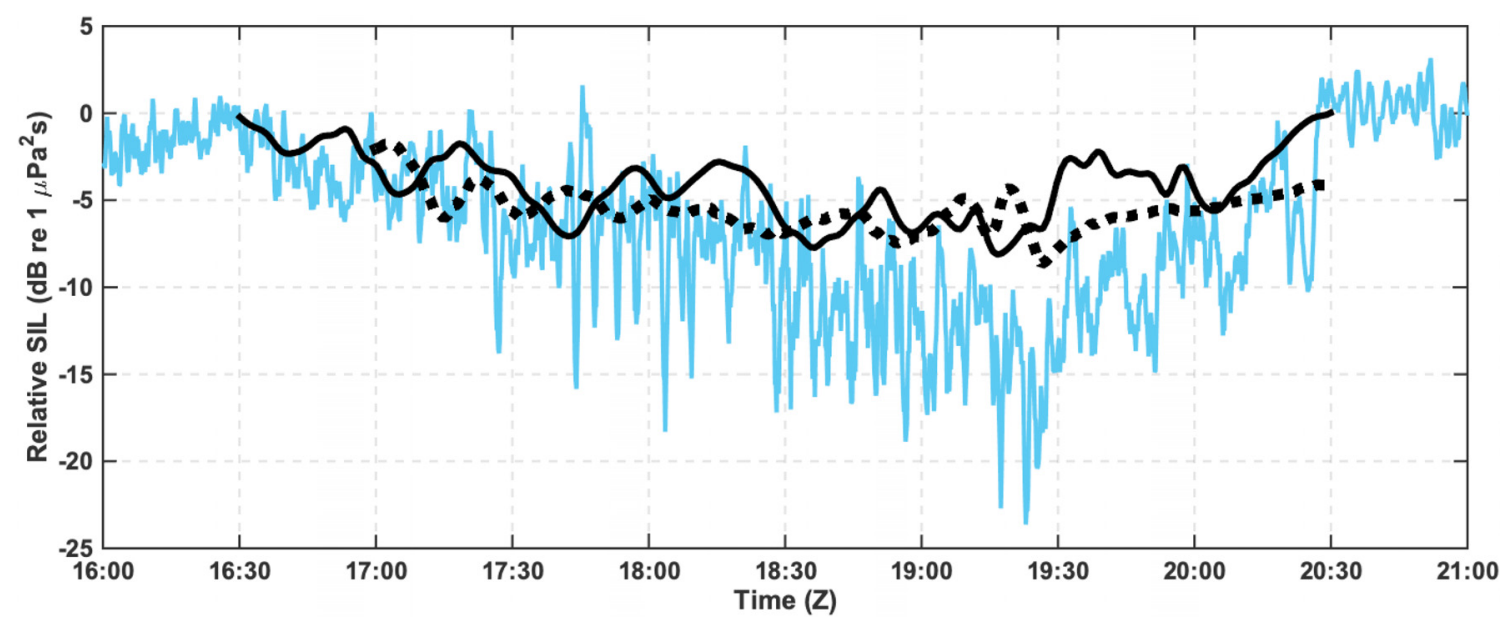

FIG. 12. (Color online) BB SIL ( $\mathrm{dB}$ re $1 \mu \mathrm{Pa}^{2} \mathrm{~s}$ ) received at the hydrophone $1 \mathrm{~m}$ above the riverbed at Station A5 as a function of time during the acoustic transmission period: observed BB SIL [thinner blue solid line, identical to Fig. 2(c)], modeled BB SIL from Bellhop2D [thick black solid line, from Reeder (2016)] and modeled BB SIL from 3DPE (thick black dotted line). 
differences (Bellhop2D used lower resolution bathymetry than 3DPE), and (b) acoustic model differences (Bellhop2D does not account for diffraction or out-of-plane propagation while 3DPE is a full-field solution.).

Significantly, during salt wedge advance, 3DPE BB SIL is overall lower than the Bellhop2D BB SIL by as much as $5 \mathrm{~dB}$, and differs by $\sim 1 \mathrm{~dB}$ at the point of maximum refraction near 1930Z. This result was verified by extensive numerical modeling to test the relative contributions to TL by factors such as salt wedge slope, salt wedge sound speed gradient, and bathymetric roughness. This additional investigation was motivated by the thought that horizontal refraction would increase with increasing slope and sound speed gradient, and that out-of-plane bottom scatter would increase with bottom roughness, particularly since the USGS $1 \mathrm{~m}$ bathymetry possesses bedforms oriented at angles oblique to the acoustic transect. Predictions by both 3DPE and $N \times 2$ DPE were obtained for various combinations of idealized salt wedge slopes (between $2^{\circ}$ and $45^{\circ}$ ), sound speed gradients (between 0.5 and $25 \mathrm{1} / \mathrm{s}$ ), and both smooth and rough bathymetry. The results of this extensive numerical experimentation demonstrated that these factors indeed create significant differences in their respective acoustic fields at specific narrowband frequencies and snapshots in time (as seen in Figs. 7-10), but these differences are averaged out when the energy is integrated over the entire $1500 \mathrm{~Hz}$ band.

Every model has its strengths and weaknesses. This ray model illustrates ray behavior and energy distribution due to refraction in the water column and out-of-plane bottom scatter, but does not account for diffraction or bottom loss. Bellhop2D accounts for vertical refraction and attenuation in the bottom, but does not account for horizontal refraction in the water column, out-of-plane scattering by the riverbed, diffraction, or geometrical dispersion (Etter, 2003). The 3DPE model is a full-physics model that can account for all loss mechanisms, limited only by environmental model accuracy and precision, as is true for all propagation models. In this case, 3DPE accounted for vertical and horizontal water column refraction, scatter by the larger-scale slope of the bottom, as well as the smaller bedforms apparent in the $1 \mathrm{~m}$ resolution bathymetric data, and bottom loss as parameterized by the geoacoustic variables; however, the water surface was modeled as horizontally planar in both the Bellhop2D and 3DPE models, so the predicted TL does not include the effect of scattering by the rough water surface.

Both the observed and modeled SIL support the interpretation that the salt wedge provides a significantly refractive waveguide, resulting in narrowband energy propagating to the east of this short $1.36 \mathrm{~km}$ acoustic transect by means of horizontal refraction in the water column and out-of-plane scattering by the bottom (Figs. 5-11). While horizontal refraction is evident in the narrowband predictions, the results indicate that horizontal refraction is not the dominant mechanism controlling broadband acoustic propagation in terms of total acoustic energy integrated over the entire $1500 \mathrm{~Hz}$ band.

Other TL mechanisms contributing to uncertainty exist which are not accounted for here; specifically, nonstationary bedforms (due to strong bottom currents during the acoustic transmission period), scattering by the rough surface, the potential presence of schools of swimbladderbearing fish, and clouds of bubbles subducted by downwelling at the tidal intrusion front. Having demonstrated here that horizontal refraction accounts for a relatively small portion of the difference between observed and modeled total acoustic energy integrated over the entire signal band, it is hypothesized that clouds of subducted bubbles are responsible for a majority of the excess, as-yet-unaccountedfor TL. This hypothesis is reasonable based upon the fact that large, persistent clouds of subducted bubbles were observed in the Columbia River during the field experiment in May 2013 (Lavery, 2015) and have been observed in the Fraser River (Baschek and Farmer, 2010), Strait of Georgia, BC (Bashek et al., 2006), James River (Marmorino and Trump, 1996), and Connecticut River (Lavery et al., 2018; Reeder et al., 2018) estuaries. Attenuation by bubble clouds have been studied via theory (Hahn, 2007) and observation (Roy et al., 1992; Hwang and Teague, 2000), demonstrating that bubble clouds attenuate acoustic signals at low-to-midfrequencies.

\section{CONCLUSIONS}

Two 3D acoustic propagation models have been employed to elucidate the variability of the acoustic field under the influence of an estuarine salt wedge in the Columbia River estuary: 3D rays and 3DPE. This study highlights the capabilities of the 3DPE model to demonstrate the spatial and temporal variability of the acoustic field in a highly complex and dynamic environment. Model results were compared to data collected during a field experiment in the Columbia River Estuary in May 2013. Ray theory demonstrates that the dominant physical mechanism controlling narrowband TL shifts from bottom scatter in a non-refractive waveguide (before the entrance of the salt wedge) to $3 \mathrm{D}$ acoustic refraction with minimal bottom interaction in a refractive waveguide (when the salt wedge occupies the acoustic transect). Observations show that the received signal energy is relatively stable before and after the passage of the salt wedge front when the acoustic path consists of a single medium (either entirely fresh water or entirely salt water), and suffers a $15 \mathrm{~dB}$ loss in the dual-media environment during salt wedge front passage. The 3DPE model predictions demonstrate that vertical and horizontal refraction in the water column and out-of-plane scattering by the bottom are clearly evident at narrowband frequencies and contribute to, but do not account for, all the observed BB TL. Future work will seek to quantify the contribution to TL by rough surface scattering and fish schools (when present), and to examine the hypothesis that a majority of the excess, unaccounted-for BB TL is due to acoustic scattering and absorption by clouds of subducted bubbles that have been observed at the tidal intrusion front.

\section{ACKNOWLEDGMENTS}

Environmental input to the acoustic models included high resolution bathymetric survey data provided by Guy Gelfenbaum (USGS), and modeled temperature and salinity 
profiles of the water column provided by Antonio Baptista, Charles Seaton, and Paul Turner at CMOP. The authors thank Derek Olson (NPS) for invaluable assistance with running the 3DPE model on NPS HPC resources. This work was supported by the Office of Naval Research.

Baptista, A. M., Seaton, C., Wilkin, M., Riseman, S., Needoba, J. A., Maier, D., Turner, P. J., Karna, T., Lopez, J. E., and Herfort, L. (2015). "Infrastructure for collaborative science and societal applications in the Columbia River estuary," Frontiers Earth Sci. 9(4), 659-682.

Baschek, B., and Farmer, D. M. (2010). "Gas bubbles as oceanographic tracers," J. Atm. Oc. Tech. 27, 241-245.

Baschek, B., Farmer, D. M., and Garrett, C. (2006). "Tidal fronts and their role in air-sea gas exchange," J. Mar. Res. 64(4), 483-515.

Bergman, P. G. (1946). "The wave equation in a medium with a variable index of refraction," J. Acoust. Soc. Am. 17, 329-333.

Chen, C.-T., and Millero, F. J. (1977). "Speed of sound in seawater at high pressures," J. Acoust. Soc. Am. 62, 1129-1135.

Day, J. H. (1980). “What is an estuary?," S. Afr. J. Sci. 76, 198.

Day, J. H. (1981). "The nature, origin and classification of estuaries," in Estuarine Ecology: With Particular Reference to Southern Africa, edited by J. H. Day and A. A. Balkema (CRC Press, Rotterdam).

Dyer, K. (1998). Estuaries: A Physical Introduction (John Wiley \& Sons, New York), Chap. 1, pp. 5-25.

Etter, P. C. (2003). Underwater Acoustic Modeling and Simulation (Spon Press, London), Chap. 4.

Feit, M. D., and Fleck, J. A., Jr. (1978). "Light propagation in graded-index fibers," Appl. Opt. 17, 3990-3998.

Gelfenbaum, G. (1983). "Suspended-sediment response to semi-diurnal and fortnightly tidal variations in a mesotidal estuary: Columbia River, USA," Marine Geol. 52, 39-57.

Gelfenbaum, G., Elias, E., Stevens, A., MacMahan, J., Reniers, A., and Sherwood, C. (2014). "Impacts of large-scale morphology and bedforms on inlet dynamics: Mouth of the Columbia River, USA," in Proceedings of the 17th Physics of Estuaries and Coastal Seas (PECS) Conference, Porto de Galinhas, Pernambuco, Brazil (October 19-24).

Gelfenbaum, G., Finlayson, D., Dartnell, P., Carlson, E., and Stevens, A. (2015). "Bathymetry and backscatter from 2013 interferometric swath bathymetry systems," Survey of Columbia River Mouth, Oregon and Washington. U.S. Geological Survey data set, http://dx.doi.org/10.5066/ F7T72FHB (Last viewed 9/3/2019).

Geyer, R. (2014). Personal communication.

Hahn, T. R. (2007). "Low frequency sound scattering from spherical assemblages of bubbles using effective medium theory," J. Acoust. Soc. Am. 122(6), 3252-3267.

Hardin, R. H., and Tappert, F. D. (1973). "Applications of the split-step Fourier method to the numerical solution of nonlinear and variable coefficient wave equations," SIAM Rev. 15, 423.
Hwang, P. A., and Teague, W. J. (2000). "Low-frequency resonant scattering of bubble clouds," J. Atmos. Oceanic Technol. 17, 847-853.

Jensen, F. B., Kuperman, W. A., Porter, M. B., and Schmidt, H. (1994). Computational Ocean Acoustics (American Institute of Physics, New York).

Karna, T., Baptista, A. M., Lopez, J. E., Turner, P. J., McNeil, C., and Sanford, T. B. (2015). "Numerical modeling of circulation in high-energy estuaries: A Columbia River estuary benchmark," Ocean Modelling 88, 54-71.

Kennish, M. J. (1986). Ecology of Estuaries, Vol. 1, Physical and Chemical Aspects (CRC Press, Inc., Boca Raton, FL), 254 pp.

Lavery, A. C. (2015). Personal communication.

Lavery, A. C., Jurisa, J. T., and Geyer, W. R. (2018). "Spectral quantification of a tidal plume from an autonomous underwater vehicle," J. Acoust. Soc. Am. 143(3), 1729.

Lin, Y.-T., Duda, T. F., and Newhall, A. E. (2013). "Three-dimensional sound propagation models using the parabolic-equation approximation and the split-step Fourier method," J. Comp. Acoust. 21, 1250018.

Marmorino, G. O., and Trump, C. L. (1996). "High-resolution measurements made across a tidal intrusion front," J. Geophys. Res. 101, 25661-25674, https://doi.org/10.1029/96JC02384.

Neal, V. T. (1972). "Physical aspects of the Columbia River and its estuary," in The Columbia River Estuary and Adjacent Ocean Waters, edited by A. T. Pruter and D. L. Alverson (University of Washington Press, Seattle, WA), pp. 19-40.

Porter, M. B. (2011). The BELLHOP Manual and User's Guide, http:// oalib.hlsresearch.com/Rays/HLS-2010-1.pdf (Last viewed October 22, 2018).

Porter, M. B., and Bucker, H. P. (1987). "Gaussian beam tracing for computing ocean acoustic fields," J. Acoust. Soc. Am. 82(4), 1349-1359.

Pritchard, D. W. (1967). "What is an estuary: Physical viewpoint," in Estuaries, edited by G. H. Lauff (American Association for the Advancement of Science, Washington), Publication No. 83, pp. 3-5.

Reeder, D. B. (2016). "Field observation of low-to-mid-frequency acoustic propagation characteristics of an estuarine salt wedge," J. Acoust. Soc. Am. 139(1), 21-29.

Reeder, D. B., Honegger, D., Joseph, J., McNeil, C., Rago, T., and Ralston, D. (2018). "Acoustic propagation at low-to-mid-frequencies in the Connecticut River,” Proc. Mtgs. Acoust. 33, 005001.

Roy, R. A., Carey, W., Nicholas, M., Schindall, J., and Crum, L. A. (1992). "Low frequency scattering from submerged bubble clouds," J. Acoust. Soc. Am. 92, 2993-2996.

Sherwood, C. R., and Creager, J. S. (1990). "Sedimentary geology of the Columbia River estuary," Progress Ocean. 25, 15-79.

Tappert, F. D. (1974). "Parabolic equation method in underwater acoustics," J. Acoust. Soc. Am. 55, S34.

Zhou, J.-X., Zhang, X.-Z., and Knobles, D. P. (2009). "Low-frequency geoacoustic model for the effective properties of sandy seabottoms," J. Acoust. Soc. Am. 125(5), 2847- 2866. 